\title{
Impact of Ixekizumab Treatment on Itch and Psoriasis Area and Severity Index in Patients with Moderate-to- Severe Plaque Psoriasis: An Integrated Analysis of Two Phase III Randomized Studies
}

\author{
Gil Yosipovitch · Adam Reich · Martin Steinhoff · Anke Beselin • \\ Toby Kent · Martin Dossenbach · Lovisa Berggren · Carsten Henneges • \\ Thomas Luger
}

Received: September 27, 2018 / Published online: November 21, 2018

(C) The Author(s) 2018

\section{ABSTRACT}

Introduction: We evaluated baseline itch and its impact on the efficacy of ixekizumab (IXE) in clearing psoriasis and improving quality-of-life measures, and we explored the relationship between itch and psoriatic skin improvement. Methods: Data were analyzed from two doubleblind, randomized, controlled phase III studies

Enhanced Digital Features To view enhanced digital features for this article go to: https://doi.org/10.6084/ m9.figshare.7241108.

Electronic supplementary material The online version of this article (https://doi.org/10.1007/s13555018-0267-9) contains supplementary material, which is available to authorized users.

G. Yosipovitch

Department of Dermatology and Cutaneous

Surgery, Itch Center, University of Miami, Miami,

FL, USA

A. Reich

Department of Dermatology, University of Rzeszow, Rzeszow, Poland

\section{Steinhoff}

Department of Dermatology \& Venereology \&

Translational Research Institute, Weill Cornell

Medicine-Qatar, Hamad Medical Corporation, Qatar

University, Doha, Qatar

M. Steinhoff

Department of Dermatology, Weill Cornell

University, New York City, NY, USA
(UNCOVER-2/3) comparing etanercept (ETN), IXE, and placebo (PBO) in patients with moderate-to-severe plaque psoriasis. Long-term analysis included UNCOVER-3 data from week 0 to week 156.

Results: At week 12, a clinically meaningful improvement in itch [Itch Numeric Rating Scale (NRS) reduction $\geq 4$ ] was seen in $70.0 \%, 88.6 \%$, and $90.8 \%$ of the IXE-treated patients in the baseline Itch NRS 4-6, 7-8, and 9-10 groups, respectively (all itch severity groups $p<0.001$ versus ETN and PBO). Also, 68.9\%, 67.1\%, and $73.6 \%$ of the IXE-treated patients in the baseline Itch NRS 4-6, 7-8, and 9-10 groups, respectively, showed an improvement of $\geq 90.0 \%$ in the Psoriatic Area and Severity Index (PASI) at week 12 as compared to the baseline (PASI 90) (all itch severity groups

T. Kent

Eli Lilly and Company UK, Hampshire, United Kingdom

M. Dossenbach

Eli Lilly Regional Operations GmbH, Vienna, Austria

A. Beselin $(\varangle) \cdot$ L. Berggren · C. Henneges Lilly Deutschland GmbH, Bad Homburg vor der Höhe, Germany

e-mail: beselin_anke@lilly.com

T. Luger

Department of Dermatology, University of Muenster, Muenster, Germany 
$p<0.001$ versus ETN and $\mathrm{PBO})$. For most patients, itch reduction preceded psoriatic plaque improvement. Sustained effects of IXE on itch and PASI were observed during 3 years of treatment.

Conclusions: Regardless of baseline itch severity, IXE treatment provided a rapid improvement in itch followed by clinically meaningful improvements in psoriasis.

Funding: Eli Lilly and Company.

Trial registration: ClinicalTrials.gov identifiers, NCT01597245 and NCT01646177.

Keywords: Itch; Ixekizumab; IL-17A; Psoriasis

\section{INTRODUCTION}

In patients with psoriasis, itch is a highly prevalent and frequently troublesome symptom with a negative impact on quality of life, including social and emotional functioning [1-4]. Despite this evidence, there may be a discrepancy between the patient's experience and the physician's evaluation of this symptom. In the Multinational Assessment of Psoriasis and Psoriatic Arthritis survey regarding physicians' perspectives on the management of psoriasis and psoriatic arthritis, $38.0 \%$ of patients with plaque psoriasis but only $7.4 \%$ of dermatologists identified itch as an important symptom [5]. The symptoms of psoriasis, including itch, are exacerbated by increased inflammation as well as by scratching. The result can be a vicious itch-scratch cycle, which may even cause new psoriasis plaques [6]. Controlling chronic itch and breaking the itch-scratch cycle can improve quality of life and reduce or even prevent future psoriasis relapses [7]. Recent research implicates a role of the nervous system in the pathophysiology of psoriasis [8]. The psoriasis-associated itch mediators, however, have yet to be identified [9].

Biologic drugs have proven effective for treating plaque psoriasis, including associated itch. Studies show that biologics targeting the cytokines tumor necrosis factor alpha, interleukin (IL)$12 /-23$, and IL-17A significantly reduce itch in patients with moderate-to-severe plaque psoriasis
[10-13]. Drugs that inhibit or attenuate the activity of IL-17A have demonstrated particular efficacy in rapidly reducing itch [7]. Ixekizumab (IXE) is a high-affinity monoclonal antibody that selectively targets IL-17A, acting as an IL-17A antagonist. IXE has been shown to be significantly more effective than etanercept (ETN) $[10,14,15]$, ustekinumab [11], methotrexate [16], and fumaric acid esters [16] in patients with moderate-to-severe plaque psoriasis.

The main objective of this post hoc exploratory analysis was to compare the short-term (12 weeks of therapy) efficacy of IXE to that of ETN based on itch severity at baseline. Further objectives were to describe how itch severity correlates with other baseline patient characteristics and to assess whether there is an association between itch improvement and psoriatic skin improvement with both short-term and long-term IXE treatment. The Itch Numeric Rating Scale (NRS) was used to determine baseline itch severity as well as to demonstrate a clinically meaningful itch improvement, defined as a reduction of 4 points or more [17]. Psoriatic skin improvement was assessed using the Psoriatic Area and Severity Index (PASI), with a focus on achieving an improvement of at least $90.0 \%$ from the baseline (PASI 90).

\section{METHODS}

\section{Study Design and Patients}

The current report assessed integrated data from two phase III multicenter, randomized, doubleblind, placebo- and ETN-controlled parallelgroup trials [UNCOVER-2 (NCT01597245) and UNCOVER-3 (NCT01646177)]. Two separate datasets were analyzed: the first comprised pooled data from UNCOVER-2 and 3 (UNCOVER-2/3) covering the first 12 weeks (induction period) of treatment; the second comprised long-term data for 3 years from the UNCOVER-3 trial.

Both trials have been published previously [10]. Briefly, eligible patients were $\geq 18$ years of age with a confirmed diagnosis of chronic plaque psoriasis for at least 6 months before 
baseline, had a PASI score of $\geq 12$ at both screening and baseline visits, had $\geq 10 \%$ body surface area (BSA) involvement, had a clinicianrated static Physician's Global Assessment Severity Index (sPGA) score of $\geq 3$, and were candidates for phototherapy and/or systemic therapy.

The protocols and informed consent forms for these studies were approved by an investigational review board at each site. The studies were conducted in accordance with the ethical principles of good clinical practice and the Declaration of Helsinki and its guidelines. Written informed consent was obtained from each patient at study entry before any study procedures.

For both the UNCOVER-2 and -3 trials, patients were randomized to treatment groups receiving $80 \mathrm{mg}$ IXE every 2 weeks (IXE Q2W; $N=736$ ) or 4 weeks (IXE Q4W; $N=733$ ) after a 160-mg starting dose, or ETN $50 \mathrm{mg}$ twice weekly (ETN; $N=740$ ), or placebo (PBO; $N=361$ ). All patients who participated in the long-term extension of the UNCOVER-3 trial were treated with IXE Q4W $(N=1274)$ beginning at week 12 . At week 60 , the dose could be increased to IXE Q2W if deemed necessary by the investigator. In the case of uptitration, patients continued to receive IXE treatment every 2 weeks until discontinuation or the end of the long-term extension period at 264 weeks (the data for week 0 to week 156 are considered in this analysis).

\section{Efficacy Endpoints}

The main efficacy endpoints for these analyses were the following: the proportions of patients achieving PASI 90; PASI scores of $\leq 1, \leq 2$, and $\leq 5 ; \geq 4$-point improvement in Itch NRS; complete resolution of itch as defined by an Itch NRS of 0 ; a Dermatology Life Quality Index (DLQI) score of 0 or 1 , indicating that the patient's psoriasis has no effect on their quality of life; and the mean change in Itch NRS. Additional analyses included the proportions of patients achieving an improvement in PASI of at least $75.0 \%$ compared to baseline (PASI 75) and complete resolution of psoriasis plaques, defined as $100.0 \%$ improvement in PASI from baseline (PASI 100). The relationship between
Itch NRS and PASI in terms of speed and order of improvement was also assessed.

\section{Statistical Analysis}

Demographic analyses were based on the intent-to-treat (ITT) population. Unless otherwise noted, short-term and long-term efficacy analyses were conducted in the ITT population with a baseline Itch NRS of $\geq 4$. Analysis of Itch NRS 0 and DLQI 0/1 included ITT patients who had a baseline Itch of NRS $\geq 1$.

Because the final approved dose was IXE Q2W for the induction period and IXE Q4W for the maintenance period, the IXE Q4W findings were not presented for the 12-week UNCOVER$2 / 3$ dataset, and only findings from the IXE Q2W followed by IXE Q4W (IXE Q2W/IXE $\mathrm{Q} 4 \mathrm{~W})$ arm are presented for the long-term dataset. Visits after uptitration to the Q2W dose were excluded from the long-term UNCOVER-3 comparisons, as this dosing is not approved for the maintenance period. Because of the re-randomization of responders at week 12 in the UNCOVER-2 study, long-term data from this trial are not included here. Analyses were performed based on subgroups of itch severity at baseline (Itch NRS 0, 1-3, 4-6, 7-8, and 9-10).

Baseline characteristics and demographics were compared between baseline itch severity groups using the Cochran-Mantel-Haenszel test stratified by study for categorical data and analysis of variance for continuous data, with treatment and study considered to be independent factors. Missing data were imputed using a nonresponder imputation method for the 12-week UNCOVER-2/3 dataset and a multiple imputations method for the long-term UNCOVER-3 dataset. The multiple imputation was a partial imputation of nonmonotone missing data (i.e., for intermittent missing data) using the Markov chain Monte Carlo method with the simple imputation model and then, for the monotone missing data, a sequential regression multiple imputation with the baseline score. Observed cases in patients who were still on the treatment were also analyzed in the longterm UNCOVER-3 dataset. For the short-term dataset binary endpoints, a logistic regression 
Table 1 Baseline patient demographics and clinical characteristics by baseline Itch NRS severity group (UNCOVER-2 and 3)

\begin{tabular}{|c|c|c|c|c|c|c|}
\hline & $\begin{array}{l}\text { Itch NRS } 0 \\
(\boldsymbol{N}=\mathbf{5 0})\end{array}$ & $\begin{array}{l}\text { Itch NRS } 1-3 \\
(N=369)\end{array}$ & $\begin{array}{l}\text { Itch NRS 4-6 } \\
(N=660)\end{array}$ & $\begin{array}{l}\text { Itch NRS 7-8 } \\
(N=930)\end{array}$ & $\begin{array}{l}\text { Itch NRS 9-10 } \\
(N=550)\end{array}$ & $p$ value \\
\hline Gender, $n(\%)$ & & & & & & $<0.001$ \\
\hline Male & $34(68.0)$ & $296(80.2)$ & $472(71.5)$ & $620(66.7)$ & $309(56.2)$ & \\
\hline Female & $16(32.0)$ & $73(19.8)$ & $188(28.5)$ & $310(33.3)$ & $241(43.8)$ & \\
\hline Race, $n(\%)$ & & & & & & 0.022 \\
\hline Asian & $4(8.0)$ & $10(2.7)$ & $18(2.7)$ & $29(3.1)$ & $17(3.1)$ & \\
\hline Black or African American & 0 & $6(1.6)$ & $13(2.0)$ & $24(2.6)$ & $27(4.9)$ & \\
\hline Caucasian & $46(92.0)$ & $348(94.8)$ & $624(94.5)$ & $856(92.3)$ & $490(89.6)$ & \\
\hline Other & 0 & $3(0.8)$ & $5(0.8)$ & $18(1.9)$ & $14(2.5)$ & \\
\hline Geographic region, $n(\%)$ & & & & & & $<0.001$ \\
\hline Europe & $31(62.0)$ & $210(56.9)$ & $342(51.8)$ & $354(38.1)$ & $159(28.9)$ & \\
\hline North America & $14(28.0)$ & $142(38.5)$ & $280(42.4)$ & $524(56.3)$ & $350(63.6)$ & \\
\hline BMI $\left(\mathrm{kg} / \mathrm{m}^{2}\right)$, mean $(\mathrm{SD})$ & $29.712(7.4257)$ & $29.050(6.1855)$ & $29.772(6.5925)$ & $30.748(6.8091)$ & $32.404(8.2554)$ & $<0.001$ \\
\hline $\begin{array}{l}\text { Baseline PSSI score, mean } \\
\text { (SD) }\end{array}$ & $15.7(12.72)$ & $18.0(12.34)$ & $19.9(14.58)$ & $20.1(14.56)$ & $21.0(15.32)$ & 0.021 \\
\hline $\begin{array}{l}\text { Baseline PPASI score, mean } \\
\quad(\mathrm{SD})\end{array}$ & $4.0(2.70)$ & $6.7(8.26)$ & $7.5(10.46)$ & $8.4(10.38)$ & $11.0(13.39)$ & 0.007 \\
\hline $\begin{array}{l}\text { Baseline Skin Pain VAS, } \\
\text { mean (SD) }\end{array}$ & $12.5(22.32)$ & $16.0(19.07)$ & $31.9(24.08)$ & $48.9(27.08)$ & $67.0(27.58)$ & $<0.001$ \\
\hline Baseline BSA, mean (SD) & $25.9(14.60)$ & $25.5(14.61)$ & $26.9(16.63)$ & $26.3(16.28)$ & $30.3(19.18)$ & $<0.001$ \\
\hline Baseline sPGA, mean (SD) & $3.4(0.49)$ & $3.4(0.54)$ & $3.5(0.56)$ & $3.6(0.60)$ & $3.7(0.64)$ & $<0.001$ \\
\hline Baseline PASI, mean (SD) & $18.7(6.35)$ & $18.5(6.11)$ & $20.0(7.55)$ & $20.2(7.71)$ & $22.3(8.81)$ & $<0.001$ \\
\hline Baseline DLQI, mean (SD) & $6.8(5.62)$ & $7.0(5.05)$ & $10.2(5.79)$ & $13.0(6.18)$ & $17.2(6.80)$ & $<0.001$ \\
\hline $\begin{array}{l}\text { Age at enrollment (years), } \\
\text { mean }(\mathrm{SD})\end{array}$ & $46.6(12.81)$ & $46.2(12.77)$ & $45.0(13.51)$ & $44.7(13.04)$ & $46.4(12.65)$ & 0.070 \\
\hline $\begin{array}{l}\text { Duration of psoriasis (years), } \\
\text { mean (SD) }\end{array}$ & $21.3(11.94)$ & $19.3(12.13)$ & $18.7(12.14)$ & $17.9(12.19)$ & $18.0(12.85)$ & 0.116 \\
\hline $\begin{array}{l}\text { Previous use of nonbiologic } \\
\text { systemic therapy, } n(\%)\end{array}$ & $23(46.0)$ & $169(45.8)$ & $292(44.2)$ & $466(50.1)$ & $262(47.6)$ & 0.178 \\
\hline $\begin{array}{l}\text { Previous use of biologic } \\
\text { systemic therapy, } n(\%)\end{array}$ & $10(20.0)$ & $51(13.8)$ & $111(16.8)$ & $176(18.9)$ & $148(26.9)$ & $<0.001$ \\
\hline Facial psoriasis, $n(\%)$ & $21(42.0)$ & $138(37.4)$ & $274(41.5)$ & $422(45.4)$ & $275(50.0)$ & 0.005 \\
\hline Scalp psoriasis, $n(\%)$ & $40(80.0)$ & $318(86.2)$ & $598(90.6)$ & $864(92.6)$ & $503(91.5)$ & $<0.001$ \\
\hline
\end{tabular}

$B M I$ body mass index, $B S A$ body surface area, $D L Q I$ Dermatology Life Quality Index, $N$ total number of patients, NRS Numeric Rating Scale, PASI Psoriasis Area and Severity Index, PPASI Palmoplantar Psoriasis Area and Severity Index, PSSI Psoriasis Scalp Severity Index, $S D$ standard deviation, $s P G A$ static Physician's Global Assessment, VAS visual analog scale 


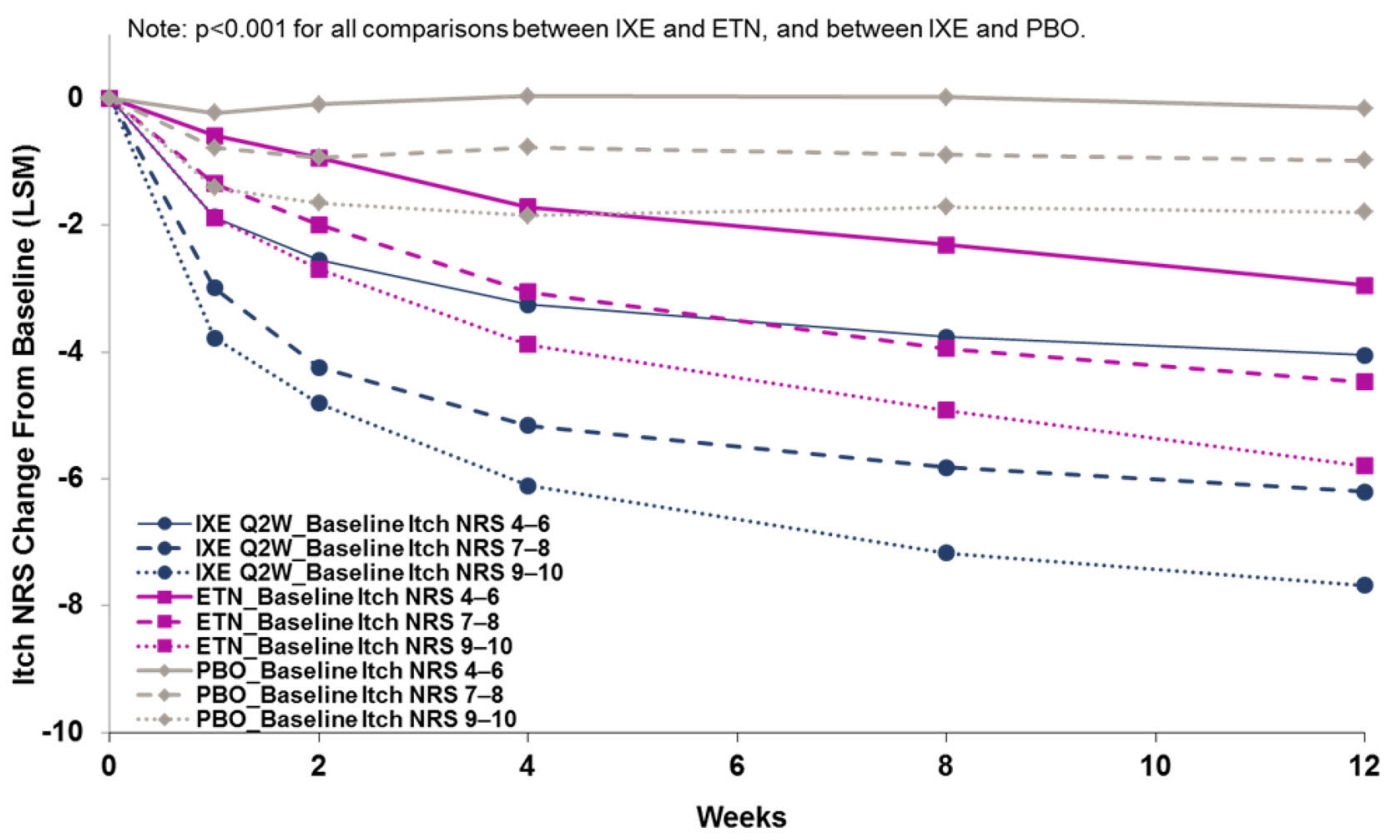

Fig. 1 Impact of baseline psoriasis-associated itch severity on the Itch Numeric Rating Scale changes from baseline during the induction period (pooled data from UNCOVER-2 and 3; LSM) in patients with psoriasisassociated Itch NRS $\geq 4$ at baseline (the LSM difference, 95\% confidence interval, and $p$ values were derived from a mixed effects model for repeated measures analysis

analysis was used, with treatment, subgroup, and the interaction of treatment with subgroup included as factors. Here the treatment-by-subgroup interaction was tested at the $10.0 \%$ significance level. The change from baseline in Itch NRS was analyzed at each study visit in the shortterm dataset using a mixed-effects model for repeated measures analysis with fixed effects (treatment, study, visit, and treatment by visit) and an unstructured variance-covariance structure. For short-term analyses, a $p$ value $<0.05$ was considered statistically significant. No correction for multiplicity was performed due to the exploratory nature of these post hoc analyses.

\section{RESULTS}

\section{Baseline Demographics and Disposition}

Among the patients in the ITT population of the integrated dataset, $1.9 \%(N=50)$ had baseline including the fixed-effects treatment, study, visit, and treatment $\times$ visit, with the variance-covariance structure set to unstructured) (intent-to-treat population). Itch NRS $4-6,7-8$, and 9-10 indicate the Itch NRS scores at baseline. ETN etanercept, IXE ixekizumab, LSM least squares mean, NRS numeric rating scale, $P B O$ placebo, Q2W every 2 weeks

Itch NRS $0,14.4 \%(N=369)$ had Itch NRS $1-3$, $25.7 \%(N=660)$ had Itch NRS $4-6,36.2 \%$ $(N=930)$ had Itch NRS $7-8$, and $21.4 \%(N=550)$ had Itch NRS 9-10. Data on baseline Itch NRS were missing for $0.4 \%(N=11)$. Table 1 displays key patient demographics and other baseline characteristics categorized by itch severity at baseline (Itch NRS 0, 1-3, 4-6, 7-8, and 9-10) for the integrated dataset. Several key characteristics and baseline variables were significantly different among the itch severity categories, including gender $(p<0.001)$, race $(p=0.022)$, geographic region $(p<0.001)$, body mass index (BMI; $p<$ 0.001), baseline Psoriasis Scalp Severity Index (PSSI; $p=0.021$ ), baseline Palmoplantar Psoriasis Area Severity Index (PPASI; $p=0.007$ ), baseline Skin Pain Visual Analog Scale (VAS; $p<0.001$ ); baseline BSA $(p<0.001)$, baseline sPGA $(p<$ $0.001)$, baseline PASI $(p<0.001)$, baseline DLQI $(p<0.001)$, previous use of biologic system therapy $(p<0.001)$, facial psoriasis $(p=0.005)$, and scalp psoriasis $(p<0.001)$. 


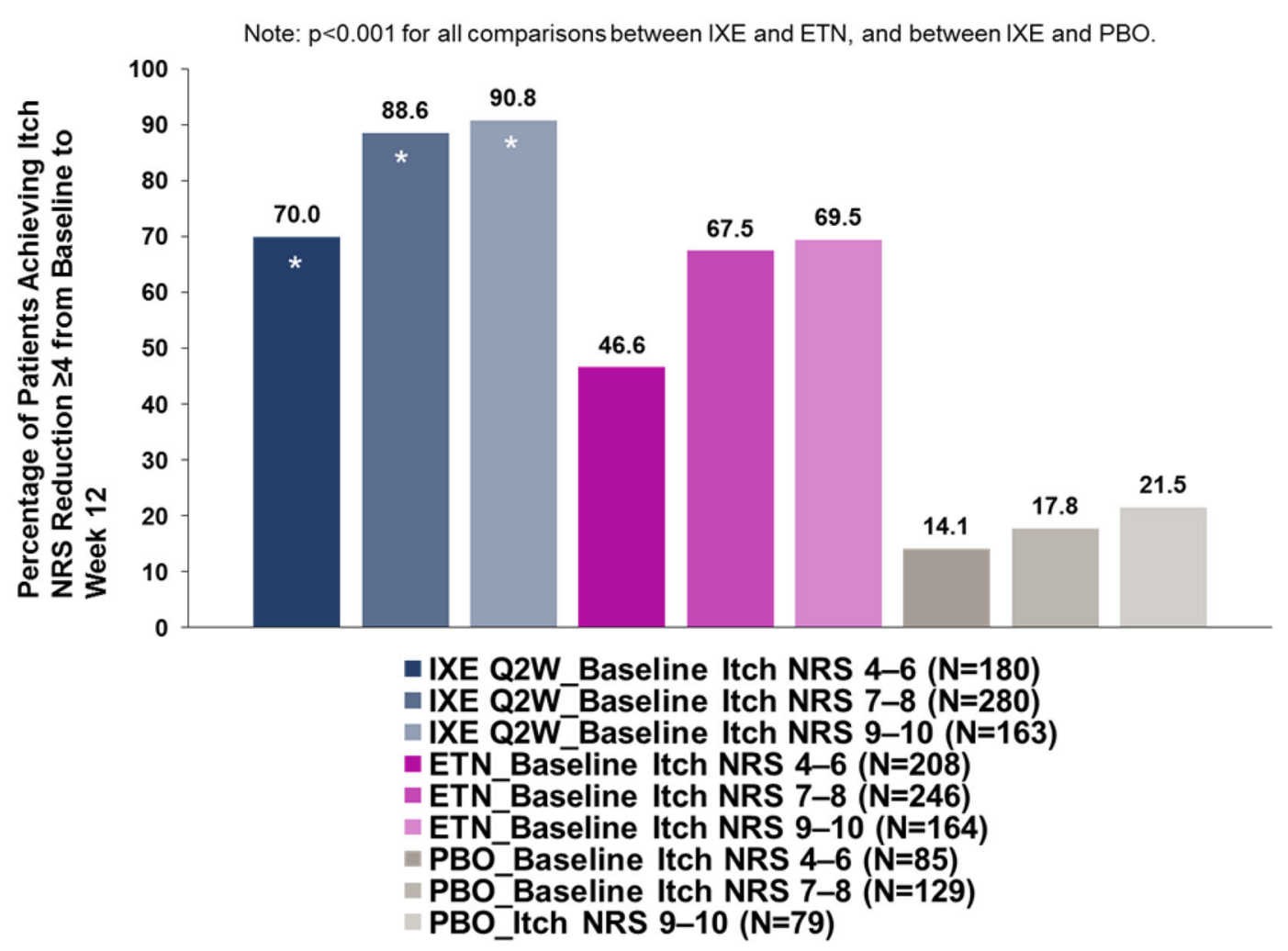

Fig. 2 Impact of baseline Itch Numeric Rating Scale severity on the response rate for a clinically meaningful improvement in itch (i.e., $\geq 4$ point reduction in Itch NRS) at week 12 in patients with psoriasis-associated Itch NRS $\geq 4$ at baseline (intent-to-treat population); treatment $p<0.001$, baseline Itch NRS category $p<0.001$,

Of note, clear numerical trends toward higher baseline Itch NRS prevalence were seen in patients from North America and those with a higher BMI, higher disease burden (PSSI, PPASI, Skin Pain VAS, sPGA, PASI), poorer DLQI, previous biologic systemic treatment, more widespread plaque psoriasis (higher BSA), and the presence of facial and/or scalp psoriasis. Baseline characteristics were well balanced across treatment groups in the ITT population among patients with baseline Itch $\mathrm{NRS} \geq 4$ (Table S1 in the Electronic supplementary material, ESM).

\section{Efficacy: Pooled Induction Period Data from UNCOVER-2 and -3}

Figure 1 shows the changes from baseline in Itch NRS during the induction period. Patients treatment $\times$ baseline Itch NRS category $(p=0.253)$, Itch NRS 4-6, 7-8, and 9-10 indicate the Itch NRS score at baseline. ETN etanercept, IXE ixekizumab, NRS Numeric Rating Scale, $P B O$ placebo, $Q 2 W$ every 2 weeks. ${ }^{*} p<0.001$ versus ETN and PBO

in the IXE Q2W arm with a baseline itch severity of Itch NRS 7-8 or 9-10 showed faster itch reduction than the ETN-treated patients. The greatest reduction was seen in patients in the IXE Q2W arm with baseline Itch NRS 9-10 (least squares mean $=-7.68$; standard error 0.21 ) at week 12. Treatment with IXE Q2W was significantly more effective than ETN treatment at all visits for all baseline Itch NRS severity subgroups $(p<0.001)$. While improvements in itch appear to be smaller in patients in the Itch NRS 4-6 subgroup treated with IXE Q2W compared to those in the Itch NRS 7-8 and 9-10 groups of the ETN arm, the maximum improvement is, by definition, 6 in the 4-6 subgroup, whereas it can be 10 in the 9-10 subgroup.

By week 12, a clinically meaningful itch improvement, defined as a reduction in Itch 


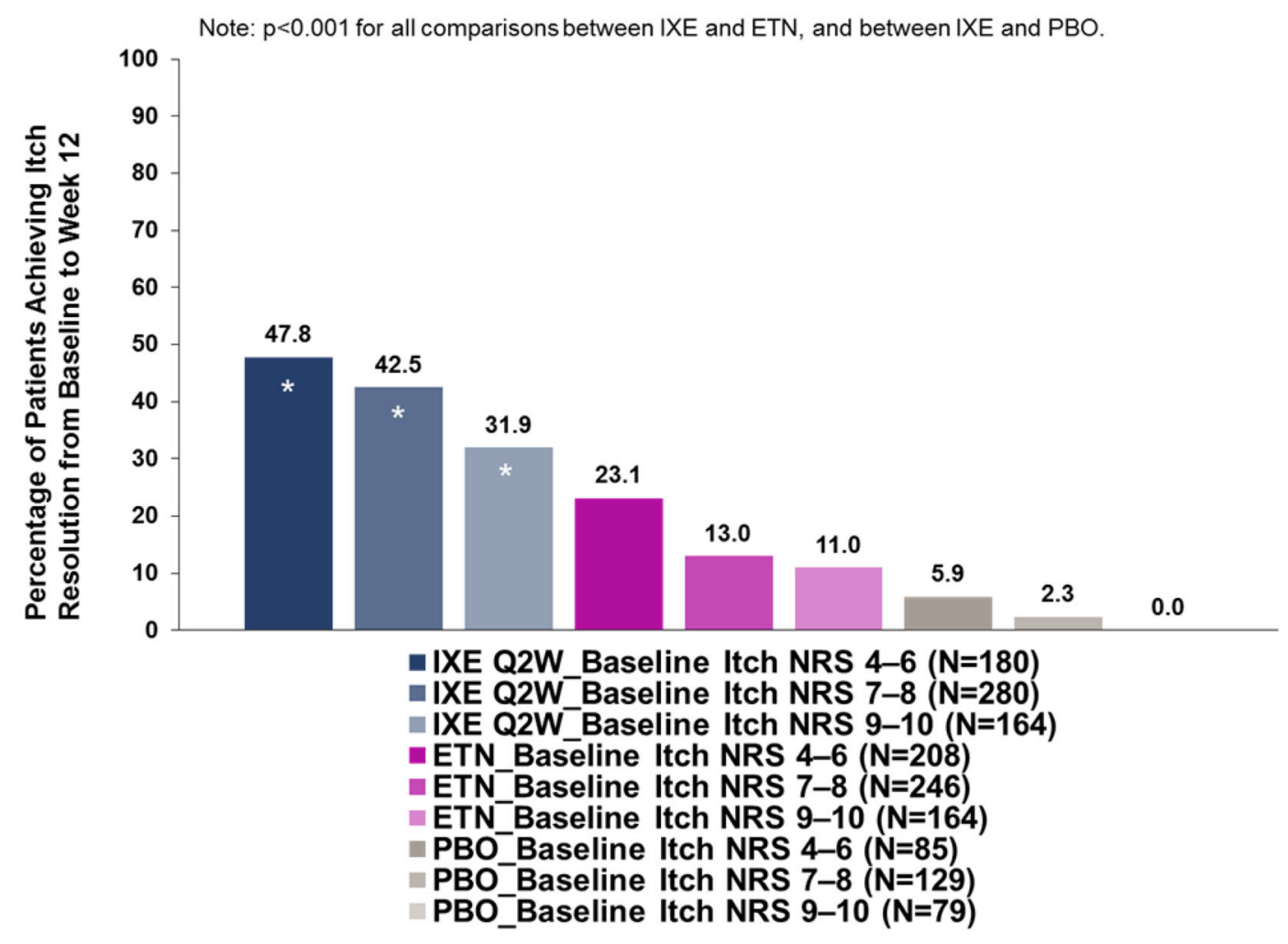

Fig. 3 Impact of baseline Itch Numeric Rating Scale severity on the response rate for complete itch resolution (i.e., Itch NRS $=0$ ) at week 12 in patients with psoriasisassociated Itch NRS $\geq 1$ at baseline (intent-to-treat population); treatment $p<0.001$, baseline Itch NRS category $p<0.001$, treatment $\times$ baseline Itch NRS category $(p=0.285)$ at the end of the induction period

NRS of $\geq 4$ points, was achieved by $70.0 \%$ (baseline Itch NRS 4-6), 88.6\% (baseline Itch NRS 7-8), and 90.8\% (baseline Itch NRS 9-10) of patients from the IXE Q2W arm, compared to $46.6 \%, 67.5 \%$, and $69.5 \%$, respectively, of patients from the ETN-treated arm (Fig. 2; $p<0.001$ for all comparisons). Total resolution of itch was attained by $47.8 \%$ (baseline Itch NRS 4-6), 42.5\% (baseline Itch NRS 7-8), and 31.9\% (baseline Itch NRS 9-10) of the patients in the IXE Q2W arm, compared to $23.1 \%, 13.0 \%$, and $11.0 \%$, respectively, of the patients in the ETNtreated arm (Fig. 3).

Across all subgroups, the IXE response rates for psoriatic skin improvement were consistently higher than the ETN rates. PASI 90 was (pooled data from UNCOVER-2 and 3; NRI), Itch NRS $4-6,7-8$, and 9-10 indicate the Itch NRS score at baseline. ETN etanercept, IXE ixekizumab, NRI nonresponder imputation, NRS Numeric Rating Scale, PBO placebo, $Q 2 W$ every 2 weeks. ${ }^{*} p<0.001$ versus ETN and $\mathrm{PBO}$

achieved by $68.9 \%$ (baseline Itch NRS 4-6), $67.1 \%$ (baseline Itch NRS 7-8), and 73.6\% (baseline Itch NRS 9-10) of patients treated with IXE Q2W, compared to $26.0 \%, 19.9 \%$, and $18.3 \%$ of patients treated with ETN (Fig. 4). PASI 75 was achieved by $87.8 \%$ (baseline Itch NRS 4-6), 88.9\% (baseline Itch NRS 7-8), and 91.4\% (baseline Itch NRS 9-10) of patients treated with IXE Q2W, compared with $55.8 \%, 43.1 \%$, and $39.0 \%$ of patients treated with ETN. PASI 100 was achieved by $43.9 \%$ (baseline Itch NRS 4-6), $38.2 \%$ (baseline Itch NRS 7-8), and 33.7\% (baseline Itch NRS 9-10) of patients treated with IXE Q2W, compared to 6.3\%, 7.3\%, and 4.9\%, respectively, of patients treated with ETN. 


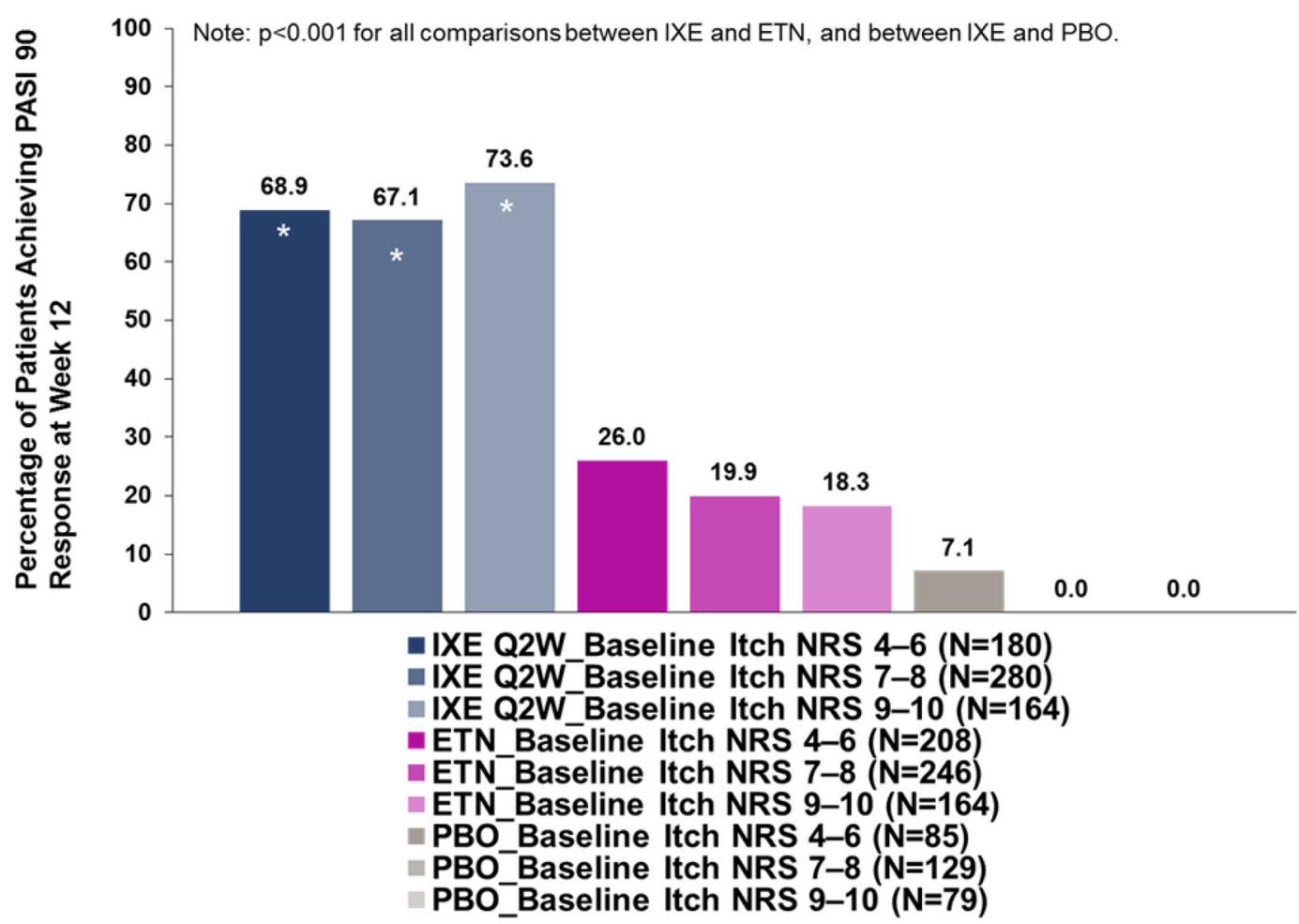

Fig. 4 Impact of baseline psoriasis-associated itch severity on the response rate for $\geq 90 \%$ improvement in Psoriasis Area and Severity Index from baseline at the end of the induction period (week 12) in patients with Itch Numeric Rating Scale $\geq 4$ at baseline (intent-to-treat population). Pooled data from UNCOVER-2 and 3; NRI; treatment $p<0.001$, baseline Itch NRS category $p=0.021$,

Similarly, IXE response rates for absolute PASI scores were consistently higher than ETN rates across all subgroups. The percentage of patients with absolute PASI $\leq 2$ is shown in Fig. 5. PASI $\leq 2$ was achieved by $71.1 \%$ (baseline Itch NRS 4-6), 70.0\% (baseline Itch NRS 7-8), and 72.4\% (baseline Itch NRS 9-10) of patients treated with IXE Q2W, compared with $30.8 \%, 22.8 \%$, and $17.1 \%$ of patients treated with ETN, respectively ( $p<0.001$ for all comparisons). Comparable results were seen for $\mathrm{PASI} \leq 1$ and PASI $\leq 5$ (Figs. S1 and S2 in the ESM,, respectively).

In some comparisons, there was a tendency for the lower Itch NRS baseline group to show greater PASI improvements. In the ETN and PBO arms, patients in the baseline Itch NRS 4-6 group appeared more likely than those in the treatment $\times$ baseline Itch NRS category $(p=0.148)$. Itch NRS 4-6, 7-8, and 9-10 indicate the Itch NRS score at baseline. ETN etanercept, IXE ixekizumab, NRI nonresponder imputation, NRS Numeric Rating Scale, PASI Psoriasis Area and Severity Index, PASI $90 \geq 90 \%$ improvement in PASI from baseline, $P B O$ placebo, Q $2 W$ every 2 weeks. ${ }^{*} p<0.001$ versus ETN and PBO

baseline Itch NRS 7-8 and 9-10 groups to achieve PASI 75/90 and absolute PASI $\leq 5 / \leq 2 /$ $\leq 1$. This pattern was also seen in the IXE Q2W arm for PASI 100 and absolute PASI $\leq 1$.

The order of onset of improvement in itch (Itch NRS $\geq 4$ ) versus psoriatic skin improvement (PASI 90) and the strength of improvement are shown in Fig. 6. Improvement in itch was achieved before PASI improvement for 65.0\% (baseline Itch NRS 4-6), 87.6\% (baseline Itch NRS 7-8), and $88.1 \%$ (baseline Itch NRS 9-10) of patients treated with IXE Q2W. Itch NRS $\geq 4$ was achieved before PASI 75 (51.4\%, $75.5 \%$, and $75.2 \%)$ as well as PASI $100(79.2 \%$, $95.5 \%$, and $98.4 \%$ ). Results were consistent with those seen for ETN-treated patients. Complete resolution of itch (Itch NRS 0) occurred before complete resolution in psoriatic skin (PASI 100) 


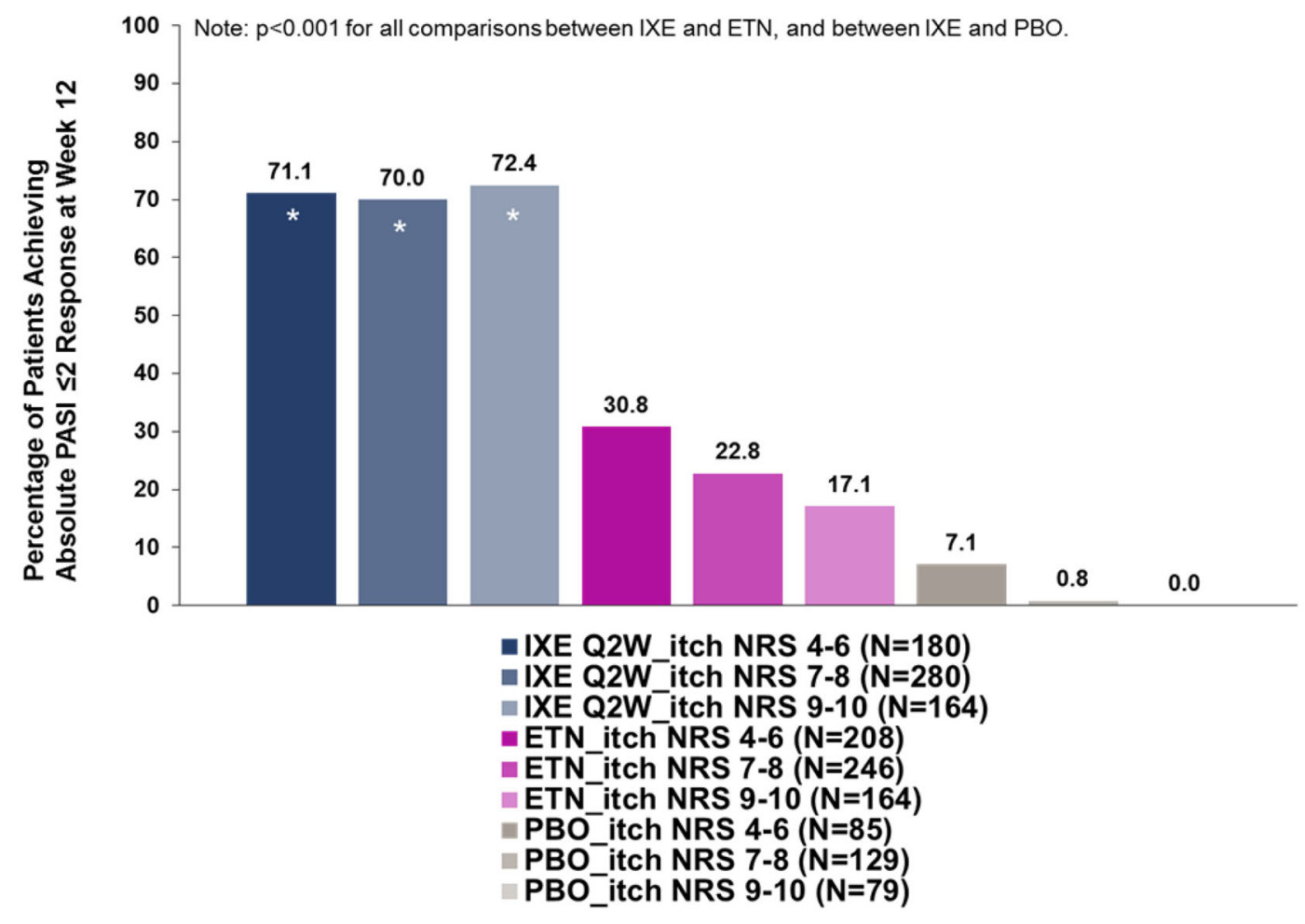

Fig. 5 Impact of baseline psoriasis-associated itch severity on the response rate for absolute Psoriasis Area and Severity Index score $\leq 2$ at week 12 in patients with Itch Numeric Rating Scale $\geq 4$ at baseline (intent-to-treat population). Treatment $p<0.001$, baseline Itch NRS category $p=0.009$, treatment $\times$ baseline Itch NRS category $(p=0.183)$ at the end of the induction period

in $30(48.4 \%)$ IXE-treated patients in the baseline Itch NRS 4-6 subgroup. Simultaneous response in terms of Itch NRS and PASI occurred in $21(33.9 \%)$ IXE-treated patients in the Itch NRS 4-6 subgroup. In patients with higher baseline itch severity, total itch resolution either preceded $(38.7 \%$ for Itch NRS 7-8, 38.9\% for Itch NRS 9-10) or occurred at the same time as $(38.7 \%$ for Itch NRS $7-8,41.7 \%$ for Itch NRS 9-10) skin clearance. At least $75.0 \%$ of the patients taking ETN reported itch resolution either before or at the same time as PASI 100 .

In a comparison of patients achieving a DLQI $0 / 1$ at week 12 based on baseline itch severity categories, a total of 123 (68.3\%) patients with baseline Itch NRS 4-6, 169 (60.4\%) patients with baseline Itch NRS 7-8, and 98 (60.1\%) patients with baseline Itch NRS 9-10 who were (pooled data from UNCOVER-2 and 3; NRI). Itch NRS 4-6, 7-8, and 9-10 indicate the Itch NRS score at baseline. ETN etanercept, IXE ixekizumab, NRI nonresponder imputation, NRS Numeric Rating Scale, $P A S I$ Psoriasis Area and Severity Index, $P B O$ placebo, $Q 2 W$ every 2 weeks. ${ }^{*} p<0.001$ versus ETN and PBO

treated with IXE Q2W reported that their psoriasis had little or no impact on their quality of life, as compared to 90 (43.3\%), 82 (33.3\%), and $43(26.2 \%)$ of the ETN-treated patients (Fig. 7, $p<0.001$ for all comparisons). There was a trend for a better quality of life of patients with lower baseline itch severity in all treatment arms.

\section{Efficacy: Long Term in UNCOVER-3}

In the long-term dataset from UNCOVER-3, $2.0 \%(N=27)$ of patients had baseline Itch NRS $0,15.6 \%(N=210)$ had baseline Itch NRS $1-3$, $26.7 \%(N=359)$ had baseline Itch NRS 4-6, $35.1 \%(N=472)$ had baseline Itch NRS 7-8, and $20.2 \%(N=272)$ had baseline Itch NRS 9-10. Data for baseline Itch NRS were missing for 


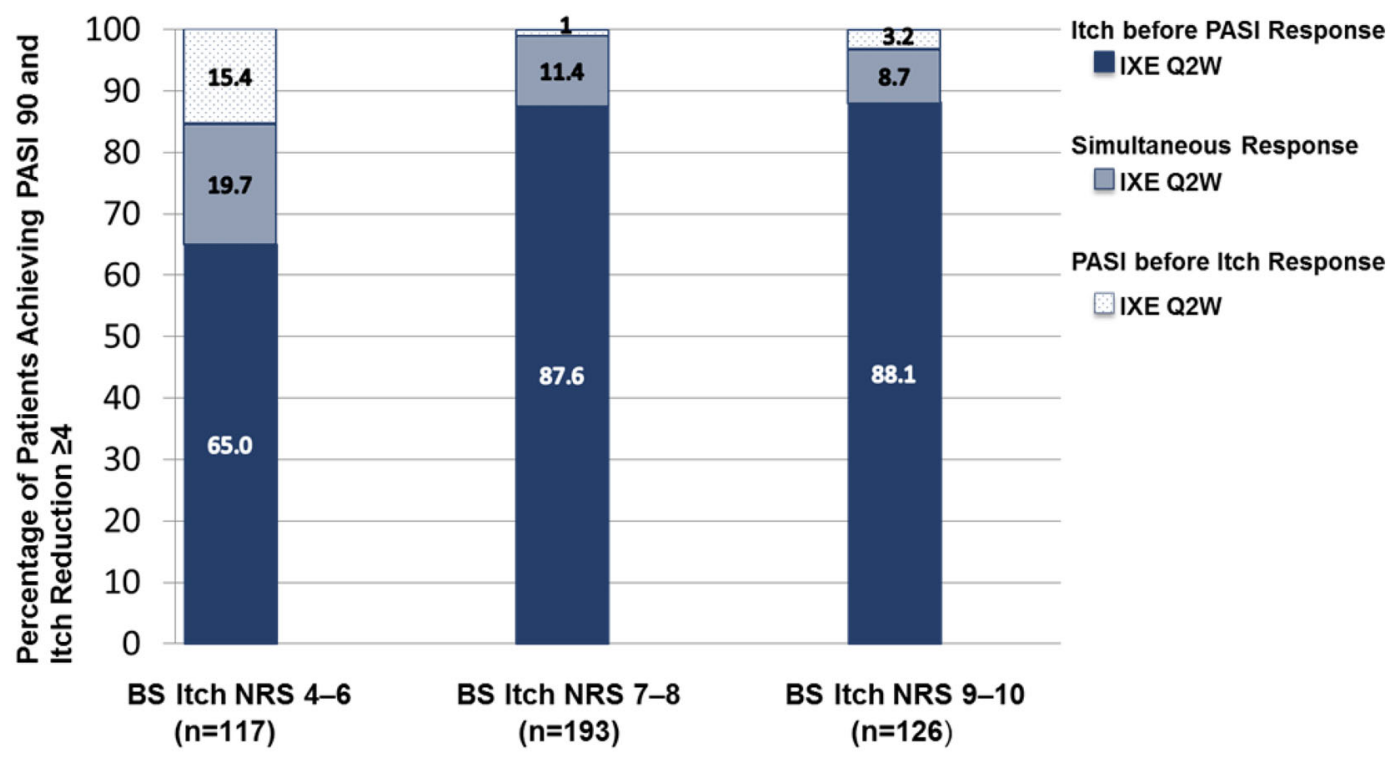

Fig. 6 Impact of baseline psoriasis-associated itch severity on order of first response based on a $\geq 90 \%$ improvement in Psoriasis Area and Severity Index from baseline and a $\geq 4$ point improvement in Itch Numeric Rating Scale from baseline at the end of the induction period (12 weeks) in patients with psoriasis-associated Itch NRS $\geq 4$ at baseline (intent-to-treat population). Pooled data from UNCOVER-2 and 3; a simultaneous response is defined as the first response in both itch and PASI

occurring at the same visit; Itch NRS 4-6, 7-8, and 9-10 indicates the Itch NRS score at baseline. BS baseline, IXE Q2W ixekizumab $80 \mathrm{mg}$ every 2 weeks, $n$ total number of patients in each subgroup to achieve both $\mathrm{a} \geq 4$ point improvement in Itch NRS and PASI 90 at some point before week 12, NRS numerical rating scale, PASI Psoriasis Area and Severity Index, PASI $90 \geq 90 \%$ improvement in PASI from baseline

$0.4 \%(N=6)$. In the long-term extension period, $15.2 \%$ of patients were uptitrated to the IXE Q2W dose. Because the final approved dose in the maintenance period was IXE Q4W, patients who uptitrated were not included in the longterm analyses.

At week 156, as determined by the multiple imputation method of analysis, $84.4 \%$ of the patients in the IXE Q2W/IXE Q4W arm achieved an Itch NRS reduction of $\geq 4$. Fewer patients with Itch NRS 4-6 at baseline achieved this clinically meaningful improvement in itch $[71.0 \% ; 95.0 \%$ confidence interval (CI) 59.9-80.8] compared with those with baseline Itch NRS 7-8 (89.1\%; CI 82.9-95.2) and baseline Itch NRS 9-10 (93.9\%; CI 86.4-99.9\%). According to comparisons of observed cases, 91.0\% of patients in the IXE Q2W/IXE Q4W arm achieved an Itch NRS reduction of $\geq 4$ at week 156. The changes in the percentage of patients with an Itch NRS reduction of $\geq 4$ over

the 3-year period as calculated by multiple imputation are shown in Fig. 8, while the corresponding data based on the observed cases are depicted in Fig. S3 of the ESM.

Results for PASI 90 over the 3-year period are shown in Fig. 9 (based on multiple imputation) and Fig. S4 of the ESM (based on the observed cases). At week 156, the percentage of the patients in the IXE Q2W/IXE Q4W arm who achieved PASI 90 was $69.0 \%$ (baseline Itch NRS 4-6), 67.4\% (baseline Itch NRS 7-8), 68.3\% (baseline Itch NRS 9-10), and 69.4\% (all baseline Itch NRS subgroups) when calculated using multiple imputation, and $85.5 \%, 83.1 \%, 85.4 \%$, and $86.5 \%$, respectively, when analyzing observed cases. PASI 75 was achieved by $86.2 \%$ (multiple imputation) and $97.1 \%$ (observed cases), and PASI 100 was achieved by $47.3 \%$ (multiple imputation) and 64.3\% (observed cases) of the patients treated with IXE Q2W/IXE 


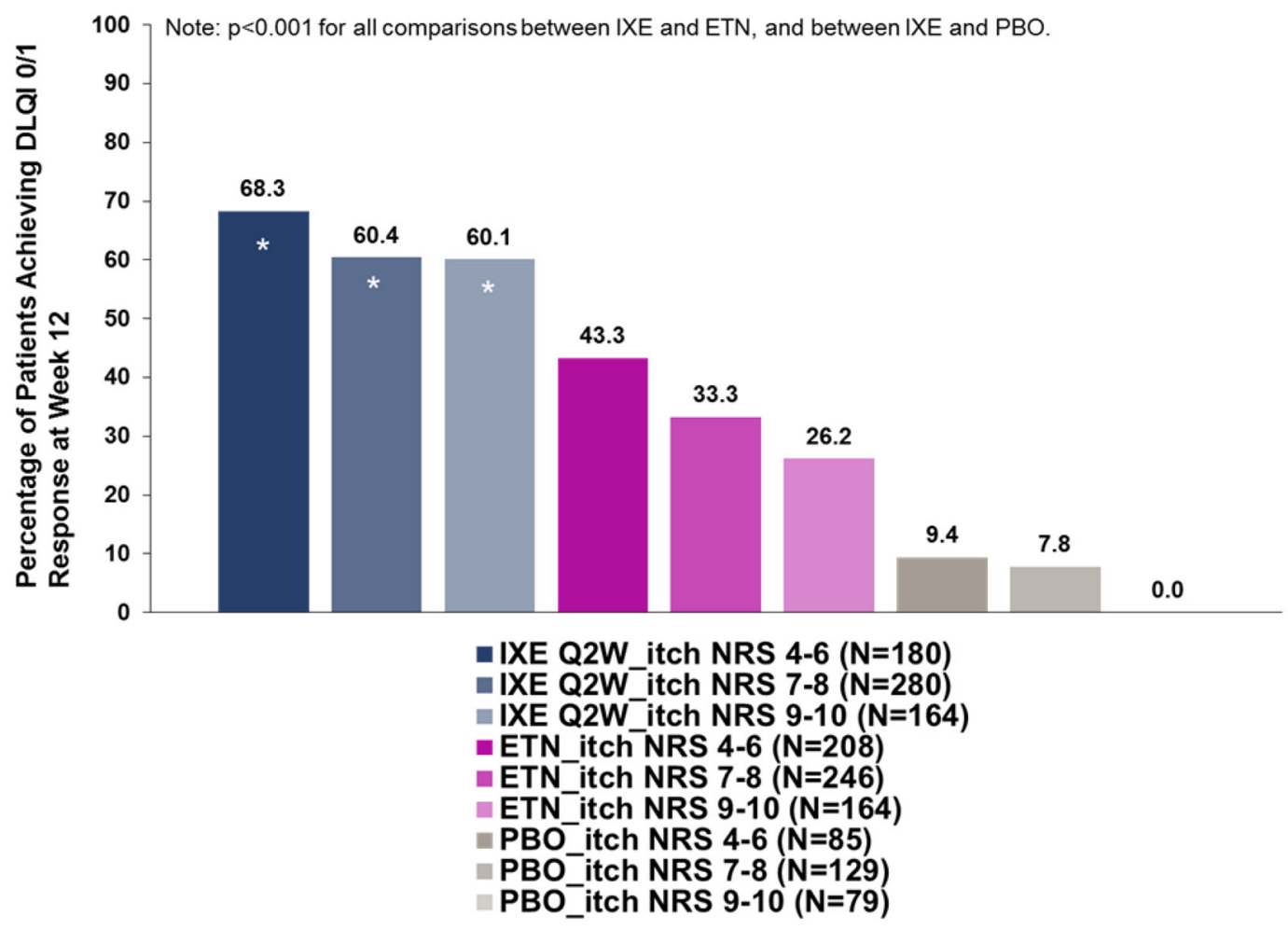

Fig. 7 Impact of baseline psoriasis-associated itch severity on response rates for Dermatology Life Quality Index scores 0 or 1 at the end of the induction period (week 12) in patients with psoriasis-associated Itch Numeric Rating Scale $\geq 1$ at baseline (intent-to-treat population). Pooled data from UNCOVER-2 and 3; NRI; treatment $p<0.001$, baseline Itch NRS category $p<0.001$,

Q4W. All PASI response outcomes were consistent across all baseline itch severity subgroups.

At week 156, an absolute PASI score of $\leq 2$ was reported by $71.8 \%$ (multiple imputation; Fig. 10) and $88.9 \%$ (observed cases; Fig. S5 of the ESM) of patients in the IXE Q2W/IXE Q4W arm. Absolute PASI $\leq 1$ was reported by $62.2 \%$ (multiple imputation) and $80.7 \%$ (observed cases) and absolute PASI $\leq 5$ was reported by 86.2\% (multiple imputation) and 97.5\% (observed cases) of patients in the IXE Q2W/IXE Q4W arm.

\section{DISCUSSION}

Our study clearly shows that IXE, a high-affinity monoclonal antibody that selectively binds IL- treatment $\times$ baseline Itch NRS category $(p=0.652)$. Itch NRS 4-6, 7-8, and 9-10 indicate the Itch NRS score at baseline. DLQI Dermatology Life Quality Index, ETN etanercept, IXE ixekizumab, NRI nonresponder imputation, NRS numeric rating scale, $P B O$ placebo, $Q 2 W$ every 2 weeks. ${ }^{*} p<0.001$ versus ETN and PBO

17A, induces a rapid improvement in itch which is followed, in most cases, by a clinically meaningful improvement in psoriasis, independent of baseline itch severity.

Greater itch severity at baseline was associated with greater psoriasis skin severity and poorer quality of life, as well as with predictors of disease severity including higher BMI and previous biologic exposure. The scalp, which is commonly reported to be an itchy area in psoriasis [18], had the highest itch severity. In short-term (12-week) comparisons with ETN, IXE provided a rapid and substantial improvement in Itch NRS. A higher percentage of IXEtreated patients achieved a clinically meaningful itch reduction (Itch NRS $\geq 4$ ) or itch resolution compared to those treated with ETN. Given that patients with psoriasis identify itch 


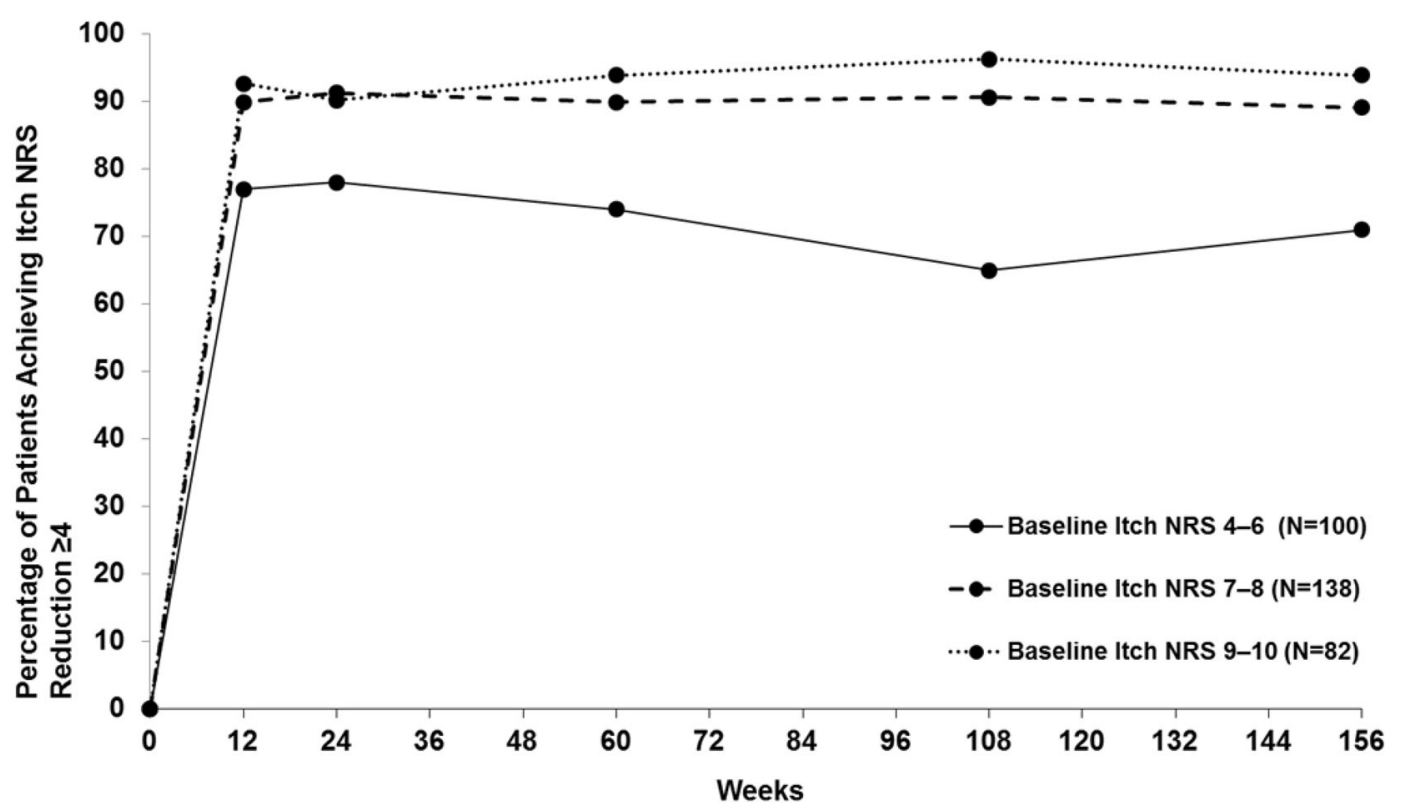

Fig. 8 Impact of baseline itch severity on the response rate for $a \geq 4$-point reduction on the Itch Numeric Rating Scale in patients with psoriasis-associated Itch NRS $\geq 4$ at baseline (intent-to-treat population) (all treatments combined, UNCOVER-3; MI). For the itch reduction, fewer patients in the Itch NRS 4-6 baseline

as the most bothersome symptom of the disease [2], finding relief from itching by achieving a clinically meaningful itch reduction or itch resolution should be considered a very relevant treatment goal.

For an indication of psoriatic skin clearance, it is important to look at both absolute PASI levels and PASI improvement as a target for treatment. Significantly higher response rates were seen with IXE than with ETN across all measures of psoriatic skin improvement at week 12 in all baseline itch groups. Thus, for patients treated with IXE, the severity of itch at baseline did not have a substantial impact on skin improvement rates in comparison to those treated with ETN. In addition, there was also no significant impact on DLQI improvement rates (i.e., patients across all itch baseline categories who were treated with IXE achieved greater quality-of-life benefits in comparison to patients treated with ETN).

For most patients, a clinically meaningful improvement in itch preceded a PASI 90 response, regardless of baseline itch severity or severity subgroup achieved clinically meaningful improvements compared to those in the Itch NRS 7-8 subgroup at weeks 24, 60, and 108 and compared to those in the Itch NRS 9-10 subgroup at weeks 12, 60, 108, and 156. Itch NRS 4-6, 7-8, and 9-10 indicate the Itch NRS score at baseline

treatment. For some efficacy outcomes (PASI 100 , absolute PASI $\geq 1$, and DLQI $0 / 1$ ), more of the patients in the lower baseline Itch NRS subgroups tended to present better results overall. Thus, one could hypothesize that an early improvement in itch may indicate subsequent skin improvement in this treatment population.

With these exploratory post hoc analyses of two phase III trials, we report the first long-term efficacy outcomes for IXE past 104 weeks. For both itch and skin improvement, IXE maintenance treatment provided a great benefit to patients with moderate-to-severe plaque psoriasis up to at least 3 years, regardless of itch severity at baseline. These analyses suggest that treatment with IXE is highly effective for up to 3 years in patients with moderate-to-severe plaque psoriasis, regardless of itch severity at baseline. Although there were some differences in the Itch NRS $\geq 4$ responses of patients with lower baseline itch severity across the 156-week period, this may be considered a ceiling effect since patients did not demonstrate 


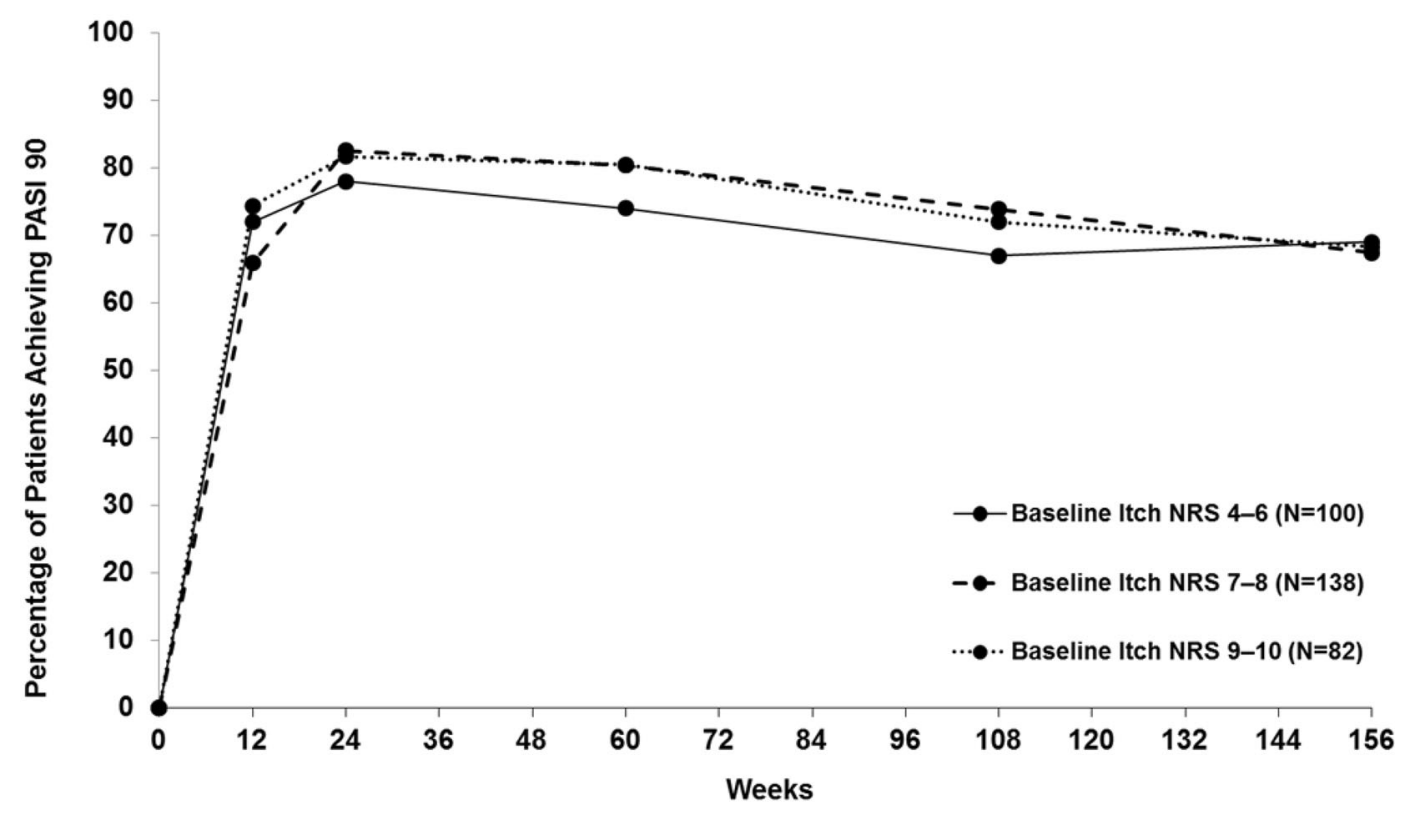

Fig. 9 Impact of baseline itch severity on the response rate for $a \geq 90 \%$ improvement in Psoriasis Area and Severity Index from baseline in patients with psoriasisassociated Itch NRS $\geq 4$ at baseline (intent-to-treat population) (all treatments combined, UNCOVER-3; MI). For itch reduction, fewer patients in the Itch NRS 4-6 baseline severity subgroup achieved clinically meaningful improvements compared to those in the Itch NRS

improvements of more than 4,5 , or 6 points, depending on their baseline score.

The results of these analyses raise further questions regarding the role of IL-17A in the pathophysiology of psoriatic itch and potential direct mechanisms for IL-17A inhibition in itch reduction. Besides its role in keratinocyte activation and proliferation, IL-17A plays a role in both central and peripheral neurogenic inflammation [7]. IL-17A stimulates keratinocytes, neurons, and glia, leading to downstream activation of the nuclear factor- $\kappa \mathrm{B}$ and mitogenactivated protein pathways, and it can enhance chemotactic signaling through synergistic effects with other cytokines, thereby recruiting more inflammatory immune cells. Elevated levels of circulating IL-17A are seen in patients with psoriasis [19] and in lesioned psoriatic skin [20]. Most dorsal root ganglion neurons express the IL-17A receptor and are excited by IL-17A $[21,22]$. While studies of IL-17A in these neurons have focused on enhanced nociception, it
7-8 subgroup at weeks 24,60 , and 108 and compared to those in the Itch NRS 9-10 subgroup at weeks 12, 60, 108, and 156. Itch NRS 4-6, 7-8, and 9-10 indicate the Itch NRS score at baseline. $M I$ multiple imputation, NRS Numeric Rating Scale, PASI Psoriasis Area and Severity Index, PASI $90 \geq 90 \%$ improvement in PASI from baseline, $P B O$ placebo, $Q 2 W$ every 2 weeks, $Q 4 W$ every 4 weeks

is possible that elevated levels of IL-17A would also enhance itch signals at the dorsal root ganglion, either directly or indirectly. Additionally, neuropeptides or proteases may induce and/or aggravate neurogenic inflammation or itch in psoriasis $[9,23]$.

The rapid response itch to IXE as compared to the speed with which it cleared psoriatic skin may therefore support the theory of a direct mechanism for the effect of IXE on itch at the peripheral level and/or central level. While the psoriatic plaques and the associated local inflammation-each of which can contribute to psoriasis itch-persist and resolve more slowly, IXE rapidly acts to block the itch signal and the experience of itch by exerting a direct effect on the IL-17/IL-17 receptor signaling pathway. This, in turn, can prevent scratching and the associated further inflammation of the skin, allowing plaques to heal and enhancing the calming action of IXE on local keratinocytes and immune cells. 


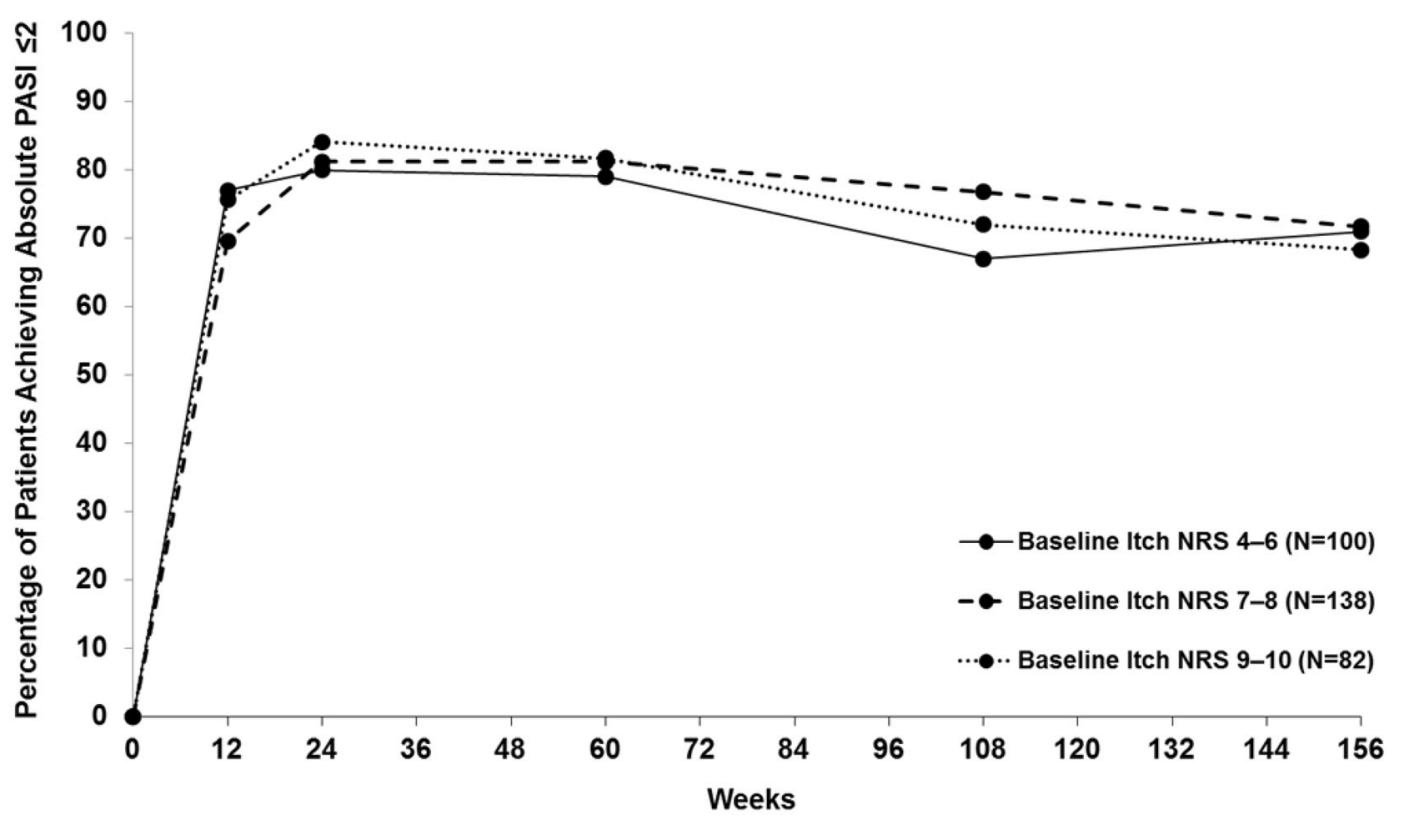

Fig. 10 Impact of baseline itch severity on the response rate for absolute PASI scores $\leq 2$ during the long-term extension period (IXE Q2W/IXE Q4W treatment) in patients with psoriasis-associated Itch NRS $\geq 4$ at baseline (intent-to-treat population) (all treatments combined, UNCOVER-3; MI). For itch reduction, fewer patients in the Itch NRS 4-6 baseline severity subgroup achieved a clinically meaningful improvement compared to those in

There are limitations to this analysis that should be considered. The main limitation is that patients were not stratified by baseline Itch NRS during randomization. The Itch NRS 7-8 group $(N=930)$ contained $95.0 \%, 60.0 \%$, $29.0 \%$, and $41.0 \%$ more patients than the Itch NRS $0(N=50), 1-3(N=369), 4-6(N=660)$, and 9-10 $(N=550)$ groups, respectively. The study is also limited by the lack of comparative data beyond week 12. Although the trend for long-term efficacy with IXE is clear, a larger population with comparative data at later time points may afford a better understanding of persistent effects attributed to baseline itch severity. Also, since the studies were initiated before IXE approval, some patients received doses that were not part of the approved dosing regimen and thus were not included in the analysis, which further reduced the number of patients included in the current study. This could potentially bias the results of the study given that these data were excluded from the the Itch NRS 7-8 subgroup at weeks 24, 60, and 108 and compared to those in the Itch NRS 9-10 subgroup at weeks 12, 60, 108, and 156. Itch NRS 4-6, 7-8, and 9-10 indicate the Itch NRS score at baseline. IXE ixekizumab, $M I$ multiple imputation, NRS Numeric Rating Scale, $P A S I$ Psoriasis Area and Severity Index, $Q 2 W$ every 2 weeks, $Q 4 W$ every 4 weeks

analysis. Further, although patients from many geographic regions were included, the study population was mostly white. Evaluation of a larger population including non-white participants with a range of itch severities would help to explain the efficacy of IXE and the impact of baseline itch in a more genetically diverse population. Lastly, PASI and itch data were only collected at discrete time points and not via a diary.

\section{CONCLUSION}

As in the previous report in which treatment with IXE was found to be significantly superior to ETN treatment [10], here we report that a higher percentage of the patients treated with IXE showed a response in terms of itch and psoriatic skin measures, independent of baseline itch severity. With IXE, substantial reductions in Itch NRS were seen within the first week 
(Fig. 1), and, in the majority of patients, clinically meaningful improvements preceded PASI 90 responses (Fig. 6). With the recent emergence of PASI 90 as a new treatment goal for psoriasis, this is of great clinical relevance. Regardless of baseline itch severity, a large number of patients treated with IXE achieved a clinically meaningful reduction in itch severity, relieving those patients from a very bothersome symptom. The fact that itch improvement preceded PASI improvement in the majority of those patients could potentially indicate subsequent skin improvement. Long-term treatment with IXE every 4 weeks provided consistent results and effective control over itch and psoriatic skin symptoms in patients with moderateto-severe plaque psoriasis.

\section{ACKNOWLEDGEMENTS}

Funding. This exploratory study was funded by Eli Lilly and Company (Lilly), Indianapolis, Indiana. Lilly also funded the article processing charges. All authors had full access to all of the data in this study and take complete responsibility for the integrity of the data and the accuracy of the data analysis.

\section{Medical Writing and Editorial Assis-} tance. The authors would like to thank Shannon E. Gardell and Angela Lorio of Syneos Health for providing writing and editorial support. This was funded by Lilly.

Authorship. All named authors meet the International Committee of Medical Journal Editors (ICMJE) criteria for authorship for this article, take responsibility for the integrity of the work as a whole, and have given their approval for this version to be published.

Disclosures. Gil Yosipovitch has served as a consultant and advisory board member of Eli Lilly and Company, Pfizer, Menlo, Trevi, Sanofi, Galderma, Sienna, Opko, Galderma, and Novartis, and has received research grants from the Leo Foundation, Pfizer, GSK, and Allergan. Adam Reich has served as a consultant, advisory board member, and/or paid speaker for, and/or has received research grants and/or honoraries for consulting and/or scientific lectures from, and/or got travel expenses reimbursed and/or participated in clinical trials sponsored by Celgene, Almirall, Leo Pharma, Eli Lilly and Company, Novartis, Menlo Pharmaceuticals, Chema Elektromet, Sun-Farm, Bioderma, and Galderma. Martin Steinhoff has served as consultant, advisory board member and/or paid speaker for, and/or has received research grants and/or honoraries for consulting and/or scientific lectures from, and/or has got travel expenses reimbursed and/or has participated in clinical trials sponsored by AbbVie, Almirall, Bayer, BMS, Eli Lilly and Company, Galderma, Janssen, Leo, Novartis, Menlo Pharmaceuticals, Pfizer, Pierre-Fabre, Sanofi, and Zymogenetics. Anke Beselin is an employee and stockholder of Eli Lilly and Company. Carsten Henneges is an employee and stockholder of Eli Lilly and Company. Martin Dossenbach is an employee and stockholder of Eli Lilly and Company. Lovisa Berggren is a contractor for Eli Lilly and Company. Toby Kent was an employee of Eli Lilly and Company during the preparation of the manuscript. Thomas Luger conducted clinical trials or received honoraria for serving as a member of the scientific advisory boards of AbbVie, Biogen-IDEC, Celgene, CERIES, Galderma, Eli Lilly and Company, Janssen-Cilag, La Roche Posay, Maruho, Meda, MSD, Mundipharma, Novartis, Pfizer, Sandoz, Sanofi-Aventis, Symrise, and Wolff.

Compliance with Ethics Guidelines. All procedures performed in studies involving human participants were in accordance with the ethical standards of the institutional and/or national research committee and with the 1964 Helsinki Declaration and its later amendments or comparable ethical standards. Informed consent was obtained from all individual participants included in the study.

Data Availability. The datasets obtained and/or analyzed during the current study are available from the corresponding author on reasonable request. 
Open Access. This article is distributed under the terms of the Creative Commons Attribution-NonCommercial 4.0 International License (http://creativecommons.org/licenses/ by-nc/4.0/), which permits any noncommercial use, distribution, and reproduction in any medium, provided you give appropriate credit to the original author(s) and the source, provide a link to the Creative Commons license, and indicate if changes were made.

\section{REFERENCES}

1. Zachariae R, Lei U, Haedersdal M, Zachariae C. Itch severity and quality of life in patients with pruritus: preliminary validity of a Danish adaptation of the Itch Severity Scale. Acta Derm Venereol. 2012;92:508-14.

2. Globe D, Bayliss MS, Harrison DJ. The impact of itch symptoms in psoriasis: results from physician interviews and patient focus groups. Health Qual Life Outcomes. 2009;7:62.

3. Reich A, Hrehorów E, Szepietowski JC. Pruritus is an important factor negatively influencing the wellbeing of psoriatic patients. Acta Derm Venereol. 2010;90:257-63.

4. Pariser D, Schenkel B, Carter C, et al. A multicenter, non-interventional study to evaluate patient-reported experiences of living with psoriasis. J Dermatol Treat. 2016;27:19-26.

5. Lebwohl MG, Kavanaugh A, Armstrong AW, et al. US perspectives in the management of psoriasis and psoriatic arthritis: patient and physician results from the population-based Multinational Assessment of Psoriasis and Psoriatic Arthritis (MAPP) survey. J Am Acad Dermatol. 2014;70:871-81.

6. Dawn A, Yosipovitch G. Treating itch in psoriasis. Dermatol Nurs. 2006;18:227-33.

7. Stull C, Grossman S, Yosipovitch G. Current and emerging therapies for itch management in psoriasis. Am J Clin Dermatol. 2016;17:617-24.

8. Riol-Blanco L, Ordovas-Montanes J, Perro M, et al. Nociceptive sensory neurons drive interleukin-23mediated psoriasiform skin inflammation. Nature. 2014;510:157-61.

9. Suárez AL, Feramisco JD, Koo J, et al. Psychoneuroimmunology of psychological stress and atopic dermatitis: pathophysiologic and therapeutic updates. Acta Derm Venereol. 2012;92:7-15.

10. Griffiths CE, Reich K, Lebwohl M, et al. Comparison of ixekizumab with etanercept or placebo in moderate-to-severe psoriasis (UNCOVER-2 and UNCOVER-3): results from two phase 3 randomised trials. Lancet. 2015;386:541-51.

11. Reich K, Pinter A, Lacour JP, et al. Comparison of ixekizumab with ustekinumab in moderate-to-severe psoriasis: 24 -week results from IXORA-S, a phase III study. Br J Dermatol. 2017;177:1014-23.

12. Kimball $A B$, Luger $T$, Gottlieb $A$, et al. Impact of ixekizumab on psoriasis itch severity and other psoriasis symptoms: results from 3 phase III psoriasis clinical trials. J Am Acad Dermatol. 2016;75:1156-61.

13. Lazzeri L, Pescitelli L, Tripo L, et al. Reduction in psoriasis related pruritus during biologic therapy. Dermatol Ther. 2017;30:e12442.

14. Leonardi CL, Blauvelt A, Sofen HL, et al. Rapid improvements in health-related quality of life and itch with ixekizumab treatment in randomized phase 3 trials: results from UNCOVER-2 and UNCOVER-3. J Eur Acad Dermatol Venereol. 2017;31:1483-90.

15. Gordon KB, Blauvelt A, Papp KA, et al. Phase 3 trials of ixekizumab in moderate-to-severe plaque psoriasis. N Engl J Med. 2016;375:345-56.

16. Reich K, Pinter A, Leutz A, et al. A 24-week, randomized, open-label comparison of ixekizumab versus fumaric acid esters and methotrexate in patients with moderate-to-severe plaque psoriasis naïve to systemic therapy. Paper presented at 26th European Academy of Dermatology and Venereology Congress; 13-17 Sep 2017; Geneva.

17. Kimball AB, Naegeli AN, Edson-Heredia E, et al. Psychometric properties of the Itch Numeric Rating Scale in patients with moderate-to-severe plaque psoriasis. Br J Dermatol. 2016;175:157-62.

18. Bin Saif GA, Ericson ME, Yosipovitch G. The itchy scalp-scratching for an explanation. Exp Dermatol. $2011 ; 20: 959-68$.

19. Takahashi H, Tsuji H, Hashimoto Y, et al. Serum cytokines and growth factor levels in Japanese patients with psoriasis. Clin Exp Dermatol. 2010;35:645-9.

20. Johansen C, Usher PA, Kjellerup RB, et al. Characterization of the interleukin-17 isoforms and receptors in lesional psoriatic skin. Br J Dermatol. 2009;160:319-24. 
21. Moynes DM, Vanner SJ, Lomax AE. Participation of interleukin $17 \mathrm{~A}$ in neuroimmune interactions. Brain Behav Immun. 2014;41:1-9.

22. Segond von Banchet G, Boettger MK, König C, et al. Neuronal IL-17 receptor upregulates TRPV4 but not TRPV1 receptors in DRG neurons and mediates mechanical but not thermal hyperalgesia. Mol Cell Neurosci. 2013;52:152-60.

23. Steinhoff M, Bienenstock J, Schmelz M, et al. Neurophysiological, neuroimmunological, and neuroendocrine basis of pruritus. J Investig Dermatol. 2006;126:1705-18. 